\title{
The Influence of Reference Acceptance and Reuse on Conversational Memory Traces.
}

ARTICLE in JOURNAL OF EXPERIMENTAL PSYCHOLOGY LEARNING MEMORY AND COGNITION · JULY 2014

Impact Factor: 2.86 · DOI: 10.1037/xIm0000036 · Source: PubMed

READS

56

2 AUTHORS:

Dominique Knutsen

University of Essex

13 PUBLICATIONS 6 CITATIONS

SEE PROFILE
Ludovic Le Bigot

Université de Poitiers

52 PUBLICATIONS 145 CITATIONS

SEE PROFILE 
This is a preprint of the manuscript entitled "The influence of reference acceptance and reuse on conversational memory traces", to be published in the Journal of Experimental

Psychology: Learning, Memory, and Cognition. Minor differences between this version and the final version of the article might be found. For the final version, please refer to the actual publication:

Knutsen, D., \& Le Bigot, L. (in press). The Influence of Reference Acceptance and Reuse on Conversational Memory Traces. Journal of Experimental Psychology: Learning, Memory, and Cognition. doi:10.1037/xlm0000036

Running head: Conversational memory traces left by dialogue management

The influence of reference acceptance and reuse on conversational memory traces

Dominique KNUTSEN and Ludovic LE BIGOT

University of Poitiers \& CNRS (CeRCA, UMR 7295), France

Corresponding author

Dominique KNUTSEN

Centre de Recherches sur la Recherche et l'Apprentissage - CeRCA - CNRS UMR 7295

MSHS Bât A5

5, rue Théodore Lefebvre

TSA 21103

F - 86073 Poitiers Cedex 9 - France

Tel: $+33(0) 549454682$

Fax: +33(0)5 49454616

E-mail: dominique.knutsen@univ-poitiers.fr or dom.knutsen@gmail.com 
Abstract

Not all pieces of information mentioned during an interaction are equally accessible in speakers' conversational memory. The current study sought to test whether two basic features of dialogue management (reference acceptance and reuse) affect reference recognition. Dyads of speakers were asked to discuss a route for an imaginary person, thus referring to the landmarks to be encountered. The results revealed that the participants' conversational memory for the references produced during the interaction depended on whether these had been reused during the interaction and by whom, along with landmark visibility during the interaction. These findings have implications for partner adaptation in dialogue, which depends in part on what speakers remember of past interactions.

Keywords: dialogue; referential communication; information accessibility; conversational memory 
The influence of reference acceptance and reuse on conversational memory traces

During dialogue, speakers often need to refer to what was said previously to produce and to interpret utterances correctly, raising the question of the nature of the memory traces left by past interactions. The link between dialogue and memory has been highlighted in studies on conversational memory. Some of the determinants of this kind of memory, such as interactional content (i.e., whether or not an utterance contains information about the speakers' intentions and attitudes towards the addressee; Keenan, MacWhinney, \& Mayhew, 1977; MacWhinney, Keenan, \& Reinke, 1982) or presence of sarcasm (Gibbs, 1986) pertain to the features of the utterances produced during the interaction; others, such as speaker gender (Baroni \& Nicolini, 1995), addressee distractibility (Pasupathi \& Hoyt, 2010), partner status (e.g., interacting with a coworker vs. interacting with one's boss; Holtgraves, Srull, \& Socall, 1989), partner familiarity (Samp \& Humphreys, 2007) or active vs. passive participation in the interaction (e.g., Baroni, D’Urso, \& Pascotto, 1991) pertain to the features of the speakers engaged in the interaction. Conversational memory also depends on the episodic cues created at the time of initial encoding (Horton, 2008; Horton \& Gerrig, 2005).

The fact that all pieces of information mentioned during an interaction are not equally likely to be remembered could have important consequences for the collaborative approach to dialogue (Clark, 1996; Clark \& Marshall, 1981; Clark \& Wilkes-Gibbs, 1986). Indeed, one of the central ideas in this approach is that any piece of information mentioned during an interaction is added to the speakers' common ground, which consists of the information that they share and are aware of sharing (Clark, 1996). Specifically, these pieces of information are added to the common ground through a joint contribution process (Clark \& Schaefer, 1989). One of the speakers starts by presenting a piece of information (e.g., a reference) for the other speaker to understand; the latter then accepts it by showing that he or she has 
understood it well enough for current purposes (Clark \& Brennan, 1991). A speaker might accept a piece of information by repeating it verbatim (in which case the information would be accepted explicitly), but he or she might also accept it by simply initiating the next relevant speech turn (in which case the information would be accepted implicitly). Accepted information can then be reused by the partners during the remainder of the interaction (Knutsen \& Le Bigot, 2012). Presentation, acceptance and reuse constitute the three steps of dialogue management. What's more, information from the common ground can be reused during subsequent interactions between the same two speakers, as common ground is partnerspecific (Brennan \& Clark, 1996; Wilkes-Gibbs \& Clark, 1992). Thus, part of Speaker A and Speaker B's common ground is based on the content of past interactions between these two speakers, which implies that they are capable of remembering what was said then.

As stated above, a number of determinants of conversational memory have already been identified, yet very little is known regarding how more basic features of dialogue management might affect conversational memory for the references produced during an interaction. For instance, the way in which a piece of information is accepted might affect its subsequent accessibility in memory. Specifically, the fact that pieces of information accepted through verbatim repetition are produced twice at the time of initial contribution could strengthen the traces they leave in the speakers' memory. However, differences at the time of acceptance might not be sufficient to account for the differences in accessibility of the pieces of information mentioned during an interaction. Indeed, accepted references can be reused between the moment when they were accepted and the end of the interaction (Knutsen \& Le Bigot, 2012); such reuse could reinforce information accessibility in memory. Since both selfand partner-produced words tend to be remembered better than non-produced words (such as, for instance, words read silently; what's more, this production benefit is enhanced when the production is self-performed; MacLeod, 2011), this should hold for both self-reused 
references (as opposed to references which were not reused by oneself) and for partner-reused references (as opposed to references which were not reused by the partner). What's more, this might hold regardless of how information was initially accepted (i.e., explicitly or nonexplicitly). Thus, reuse might have a greater influence than acceptance on conversational memory for the references produced during an interaction.

The purpose of the current study is to determine whether the strength of the memory traces left by past interactions depends on how references were accepted or on whether these references were reused (and by whom). A recognition test was used to assess participants' memory for the references mentioned during an interactive route description task. Two hypotheses were tested. First, the Acceptance Hypothesis was that references accepted through verbatim repetition are recognized better than references accepted through other means. Second, the Reuse Hypothesis was that references reused during the interaction (either by oneself or by the other person) are recognized better than non-reused ones.

\section{Method}

\section{Overview of the experiment and rationale}

In an experiment, dyads of participants interacted to perform a route description task. Each participant was given a map in order to do this; they both referred to the landmarks shown on the maps as they interacted. Although the maps represented the same area, only part of the landmarks were shown on both maps; others were only shown on one of the maps. Thus, from each participant's point of view, this experiment involved referring to privileged-to-self landmarks (which were only shown on one's own map), to shared landmarks (which were shown on both maps) and to privileged-to-other landmarks (which were only shown on one's partner's map). The purpose of this was to manipulate referent visibility from each participant's point of view, thus allowing us to determine whether the influence of acceptance 
and/or reuse on reference recognition depended on whether the corresponding referents were visible or not during the interaction (see Knutsen \& Le Bigot, 2012, for a similar method). At the end of the interaction, the participants performed an individual recognition test during which they were asked to identify the references mentioned during the interaction.

\section{Participants}

Twenty-eight dyads of native French speakers (18-25 years, 11 male) signed an informed consent form before taking part in the experiment for course credit.

\section{Apparatus}

The participants were recorded using two microphones connected to a digital recorder.

\section{Materials}

A map including 90 landmarks (24 buildings, nine squares and 57 street names) was used to build the stimuli used in this experiment. The 90 landmarks were randomly divided into three blocks (B1, B2 and B3), each including eight buildings, three squares and 19 street names. Three maps were then created, each including two blocks of landmarks. Within each dyad, each participant was given a different map (see Figure 1). For instance, Participant A would be given map B1B2 and Participant B would be given map B2B3; thus, B1 landmarks would be privileged to A (i.e., privileged-to-self from A's point of view and privileged-to-other from B's point of view), B2 landmarks would be shared and B3 landmarks would be privileged to B (i.e., privileged-to-other from A's point of view and privileged-to-self from B's point of view). Block distribution within dyads was counterbalanced across dyads. 
Paper booklets were prepared for each participant to use during the recognition test. Each booklet included all landmarks shown on at least one of the two maps (i.e., the three blocks of target landmarks), regardless of whether or not these had actually been mentioned during the interaction. The landmark labels were identical to those shown on the maps during the dialogue phase (e.g., "hôpital" ("hospital”), "place Laganne” ("Laganne Square”), "rue Jean Jaurès” (“Jean Jaurès Street”)). It also included an additional block of 30 new landmarks (eight buildings, three squares and 19 street names) which were not shown on the maps used during the dialogue phase and which served as distractors (the purpose of these was to increase the difficulty of the recognition test). The status of these new landmarks was not rotated across participants or dyads (i.e., these 30 landmarks were always new to both participants). Thus, from each participant's point of view, the booklet included $25 \%$ of entirely new landmarks, $25 \%$ of privileged-to-other landmarks which could potentially have been mentioned during the dialogue phase, but which were not shown on one's own map (note that in cases where these had not been mentioned, these landmark names were in fact "new" to the participant) and 50\% of shared and privileged-to-self landmarks which could potentially have been mentioned during the dialogue phase and which were shown on one's own map. The booklets were divided into three subsections (buildings, squares and street names); subsection presentation order was counterbalanced across dyads. Landmark names were listed in alphabetical order in each subsection. In front of each landmark name, a box could be ticked if the participant believed that it had been mentioned during the interaction.

\section{Task and procedure}

The participants performed the experiment in pairs. They sat in the experimental room, each facing a different wall of the room, so that they could not see each other. The purpose of this 
was to prevent them from using non-linguistic cues such as facial expressions to communicate during the experiment.

The participants' task consisted in discussing a route for a person who had no previous knowledge of the town represented on the maps. They knew that the landmarks differed across maps, but they did not know which were shared and which were not. They were told that all landmarks could be included in the route, even those which were not shown on both maps. They were informed that they would have to write out individually (using a pen and paper) the route chosen after the end of the interaction (this was to force them to pay attention to what was being said during the interaction), but they did not know in advance that they would have to perform a recognition test.

During the first phase of the experiment (dialogue phase), the participants had a maximum of 15 minutes to agree on a return tourist route between Point $\mathrm{A}$ and Point $\mathrm{B}$, which were shown on both maps. The participants performed the second phase of the experiment (recognition test phase) immediately after the dialogue phase. During this phase, the participants were each given a booklet and a pen and were asked to identify which landmarks had been mentioned during the interaction. The participants performed this part of the experiment at their own pace (there was no time limit) and could go back and change their responses if necessary. They could not communicate nor have access to the maps during this phase.

\section{Data coding and experimental design}

The dialogues between the participants were transcribed; the references produced by the participants were coded for presentation, acceptance and reuse (see Appendix A).

Four IVs were used: Landmark Status (Privileged-to-self, Privileged-to-other; Shared), Acceptance Type (Verbatim, Anaphoric, Non-explicit), Reuse by Self (Reused by self, Non- 
reused by self) and Reuse by Other (Reused by other, Non-reused by other). The basic analysis unit was always the participant rather than the dyad.

\section{Results}

A total of 1042 references were presented during the dialogue phase (each speaker presented $18.61(S D=5.12)$ references on average $)$. Among these, 416 were shared and 626 were privileged to one of the speakers. Regarding acceptance, 222 references were accepted through verbatim repetition, 664 were accepted anaphorically and the remaining 156 were accepted non-explicitly.

The participants reused a total of 938 references during the dialogue phase. (Recall that a reference could be reused by more than one speaker; 938 references being reused does not necessarily mean that almost all presented references were reused, as some might have been reused by two participants in a dyad: this would have counted as two separate reuses). Among these, 473 were shared, 339 were privileged-to-self and 126 were privileged-to-other. Regarding acceptance, 217 references had been accepted through verbatim repetition, 605 had been accepted anaphorically and the remaining 116 had been accepted non-explicitly.

Before performing the statistical analysis per se, we examined the participants' overall performance in the recognition test. The participants' capacity to reject the references which had not been mentioned during the dialogue phase was examined first. Regarding the references to landmarks which were actually shown on at least one of the maps during the dialogue phase, Table 1 shows that the false alarm rate was fairly low (i.e., it varied between $2.09 \%$ and $5.31 \%$ ). Regarding the references to new, distractor landmarks, the false alarm rate was even lower (i.e., 1.37\%). This false alarm rate could not be used to estimate the participants' overall tendency to say that a landmark had been mentioned, as the status of these landmarks was not counterbalanced across participants or dyads (i.e., the same 30 
landmarks were always new to the participants; something about these landmarks might have made them easier to reject than the other landmarks). However, privileged-to-other landmarks which were never mentioned during the interaction were entirely new to one of the participants in the dyad during the recognition test; since the status of these landmarks was counterbalanced across participants and dyads, the false alarm rate for these landmarks could be used as a baseline in this experiment. The results revealed that $2.13 \%$ of these were mistakenly identified as having been mentioned during the dialogue phase (precisely, a total of 1054 privileged-to-other landmarks were never referred to during the dialogue phase; among these, 22 were mistakenly identified as having been mentioned). This suggests that the overall false alarm rate was fairly low in this experiment.

Second, we looked at whether the participants were capable of correctly identifying the references which had been mentioned during the dialogue phase. The participants' responses were correct $63.05 \%$ of the time. This could mean two different things: it could mean either that the participants had a bias toward answering "no" to the landmark names in the list, or that answering "yes" to landmark names which had actually been mentioned during the interaction was somewhat more difficult than rejecting unmentioned references. In any event, the results suggest that the participants' capacity to correctly recognize these references depended on reference status: the statistical analysis reported hereafter was conducted to investigate these differences further.

Insert Table 1 around here

A logistic mixed model was used to analyze the data. Such models can be used to account for the nesting of participants in groups in cases where the outcome variable is binary (see Barr, Levy, Scheepers, \& Tily, 2013; Jaeger, 2008). This analysis was conducted using 
the GLIMMIX procedure in SAS 9.4. More detail about the random effects structure used can be found in Appendix B. As the number of observations varied across participants, the Satterthwaite correction was applied (Keselman, Algina, Kowalchuk, \& Wolfinger, 1999). Only the data corresponding to the references which had actually been presented during the interaction were analyzed.

The binary dependent variable was whether a reference received a "mentioned" judgment (which would represent a correct response) or an "unmentioned" judgment (which would represent an incorrect response). Main effects were systematically included in the models; interactions were only included if they reached statistical significance. Accordingly, the model included Landmark Status, Acceptance Type, Reuse by Self, Reuse by Other and the interaction between Reuse by Self and Reuse by Other as fixed effects. By-participant and by-item random intercepts and by-participant random slopes corresponding to Acceptance Type and Reuse by Other were included. The results are reported in Table 2; the tests of fixed effects and the model parameters are reported in Table 3.

Insert Table 2 around here

Insert Table 3 around here

Landmark Status, Reuse by Self, Reuse by Other and the interaction between Reuse by Self and Reuse by Other significantly predicted reference recognition (Table 3). In contrast, Acceptance Type failed to reach statistical significance, even though one of the corresponding parameter estimates comparison yielded a significant difference in the baseline modalities of the other factors (i.e., verbatim vs. non-explicit). 
Regarding Landmark Status, references to privileged-to-other landmarks were significantly less likely to be recognized than references to shared landmarks, $O R=.49, C I .95$ $=.38, .64$; in contrast, references to privileged-to-self landmarks were significantly more likely to be recognized than references to shared landmarks, $O R=1.38, C I_{.95}=1.05,1.81$. These results show that referent visibility during the dialogue phase affected the subsequent accessibility in memory of the corresponding references. Indeed, references to landmarks which were visible to the participants during the dialogue phase (privileged-to-self and shared) were recognized better than references to landmarks which were not visible during the dialogue phase (privileged-to-other). This extends Knutsen and Le Bigot's (2012) finding that referent visibility affects reference reuse: it also has a longer-term influence on the speakers' conversational memory for the references produced during the interaction. Moreover, the results suggest that references to privileged-to-self landmarks were recognized better than references to shared landmarks. One possible explanation is that references to privileged-toself landmarks tend to be reused more often than references to shared landmarks, thus reinforcing the corresponding memory traces.

What's more, two main effects corresponding to Reuse by Self and Reuse by Other were found. As predicted, self-reused references were recognized better than non-self-reused ones, and partner-reused references were recognized better than non-partner-reused ones, respectively $O R=2.61, C I_{.95}=2.05,3.33$ and $O R=2.64, C I_{.95}=2.00,3.48$. Furthermore, a significant interaction between Reuse by self and Reuse by other was found (see Figure 2). This interaction revealed that the participants' tendency to recognize better the references which had been reused by their partner than the references which had not been reused by their partner was smaller in cases where they had also reused these references themselves (compared with cases where they had not reused these references themselves), $O R=0.44$. Consequently, the references which had been reused by both participants were recognized 
better than the other references (i.e., the references which had been reused by one participant only and the references which had never been reused); the references which had been reused by neither participant were recognized less well than the other references (i.e., the references which had been reused by both participants and the references which had been reused by one participant only).

Insert Figure 2 around here

Finally, in this model, the influence of Acceptance Type failed to reach statistical significance. This lack of a significant difference does not necessarily mean that Acceptance Type does not affect conversational memory for the references produced during the interaction; however, it does suggest that its influence is minimized when reuse (along with referent visibility) is taken into account, in line with the Reuse Hypothesis.

\section{Discussion}

This study sought to determine how acceptance and reuse shape speakers' conversational memory for the references produced during an interaction. The statistical analysis performed showed no significant evidence that the references accepted through verbatim repetition were recognized better than the references accepted through other means, thus suggesting that reuse had a stronger influence than acceptance on the participants' performance in the recognition test. Specifically, the accessibility in memory of self-reused references was greater than that of non-self-reused references; the accessibility of the latter could then be enhanced through partner reuse. In addition, partner-reused references were also more readily accessible than non-partner-reused ones. These results imply that it is the reuse that speakers make of references, rather than the way in which these were accepted, that shapes conversational 
memory for references produced during an interaction. This is in line not only with the Reuse Hypothesis, but also with MacLeod's (2011) finding that word accessibility in memory is enhanced by both self- and partner-production. The lack of a significant interaction between Landmark Status and the other IVs prevents us from concluding that the influence of reuse on conversational memory for the references produced during an interaction depends on referent visibility, although references to non-visible referents tend to leave weaker memory traces than references to visible ones.

These findings raise the question of the nature of the memory representations examined in this study. One possibility is that episodes corresponding to reference reuse are encoded separately from episodes corresponding to presentation and acceptance (even though these might concern the same references), as both episodes are separated by several speech turns. The availability of two different episodes (rather than just one) might increase the accessibility in memory of the corresponding reference, which could help explain why reused references were recognized better than non-reused ones in this study. This could also help explain why the references reused by both participants were recognized better than the references reused by one participant only. Reuse by self and reuse by other might also be encoded separately, thus again increasing the number of episodes associated with the references reused by the two dialogue partners. However, an alternative explanation is that the same reuse memory episode is reinforced through repeated reuse by both partners. Of course, these propositions are only provisional, as they could not be tested directly in this study; future work should be directed towards addressing the possibilities developed here. For instance, whether reuse by self and reuse by other are encoded separately might depend on the number of speech turns occurring between them.

In any event, these results shed light on the role of self- and partner-production in conversational memory, as the evidence on this point is somewhat mixed (see Hjelmquist, 
1984; Jarvella \& Collas, 1974, who showed that self-produced information is remembered better than partner-produced information; see also Stafford, Burggraf, \& Sharkey, 1987; Stafford \& Daly, 1984, who reported the contrary). Specifically, they show that both self- and partner-production contribute to shaping conversational memory for the references produced during an interaction, as partner-production reinforces the accessibility in memory of information which did not benefit from the self-production benefit. Moreover, the fact that the references which had been reused by both partners were more readily accessible in memory than any other kind of reference suggests that individual and collective reuse have a different influence on conversational memory. These findings corroborate the idea that "retelling" (that is, having the opportunity to mention an encoded piece of information again between the moment when it was initially encoded and the moment when memory is assessed) can affect the accessibility in memory of previously encoded information (see Hirst \& Echterhoff, 2012; Marsh, 2007), as reference accessibility in memory depended less on reference initial encoding (i.e., presentation and acceptance) and more on what happened between initial encoding and the recognition test (i.e., reuse) in this study. Moreover, the current results reveal that the influence of retelling during dialogue on information accessibility depends on whether the retelling is performed by one speaker alone, or by both speakers together. These findings are also in line with the more general idea that collaboration has an influence on memory performance (Andersson \& Rönnberg, 1997; Barber, Rajaram, \& Aron, 2010; Rajaram, 2011).

The findings reported here have important implications for the collaborative approach to dialogue (e.g., Clark, 1996), as this approach states that the content of past interactions can be used in subsequent interactions between the same dialogue partners. Indeed, speakers can resort to their conversational memory for references produced during past interactions to assess common ground and to produce partner-adapted utterances, reasoning that a reference 
which was successfully understood earlier during the current interaction or during past interactions should be understood again (Brennan \& Clark, 1996; Clark \& Marshall, 1981). The current study sheds light on the memory traces available to each speaker to do so, suggesting that the strength of such traces depends on whether these were reused and by whom during the initial interaction. We thus suggest that in contrast to the common ground based on the information mentioned earlier during the current interaction, which is built in two steps (presentation and acceptance; Clark \& Schaefer, 1989), the common ground based on the content of past interactions is built in three phases: presentation, acceptance and reuse. Individual reuse (i.e., reuse by one speaker only) is sufficient to make references more readily accessible than if they had only been presented and accepted. Indeed, when Speaker A reuses a reference, it becomes readily accessible not only to A (by virtue of self-reuse), but also to B (by virtue of partner-reuse). However, collective reuse has an even greater influence on both speakers' conversational memory.

In conclusion, the current study supports the idea that conversational memory mainly depends on whether a reference was reused and by whom during the interaction; it also depends on referent visibility. These findings shed light on the idea that self- and partnerproduction jointly contribute to conversational memory; they also shed light on the nature of the memory traces available for speakers to use to assess common ground in subsequent interactions. 


\section{Acknowledgments}

This work was conducted as part of the first author's $\mathrm{PhD}$, which is supported by the Direction Générale de l'Armement and Région Poitou-Charentes. The authors would like to thank the reviewers for their helpful comments and advice on the previous versions of this manuscript. 


\section{References}

(Note: The reference marked with an asterisk indicates work cited in the Appendices.)

Andersson, J., \& Rönnberg, J. (1997). Cued memory collaboration: Effects of friendship and type of retrieval cue. European Journal of Cognitive Psychology, 9, 273-287. doi: $10.1080 / 713752558$

Barber, S. J., Rajaram, S., \& Aron, A. (2010). When two is too many: Collaborative encoding impairs memory. Memory \& Cognition, 38, 255-264. doi:10.3758/MC.38.3.255

Baroni, M. R., D’Urso, V., \& Pascotto, M. (1991). Memory for dialogue in different modes of interactions. Pragmatics, 1, 453-464.

Baroni, M. R., \& Nicolini, C. (1995). Natural conversations in males and females:

Conversational styles, content recall and quality of interaction. Pragmatics, 5, 407426.

Barr, D. J., Levy, R., Scheepers, C., \& Tily, H .J. (2013). Random effects structure for confirmatory hypothesis testing: Keep it maximal. Journal of Memory and Language, 68, 255-278. doi:10.1016/j.jml.2012.11.001

Brennan, S. E., \& Clark, H. H. (1996). Conceptual pacts and lexical choice in conversation. Journal of Experimental Psychology: Learning, Memory and Cognition, 22, 14821493. doi:10.1037/0278-7393.22.6.1482

Clark, H. H., \& Brennan, S. E. (1991). Grounding in communication. In L. B. Resnick, J. M. Levine, \& S. D. Teasley (Eds.). Perspectives on socially shared cognition (pp. 127149). Washington: APA Books.

Clark, H. H., \& Marshall, C. R. (1981). Definite reference and mutual knowledge. In A. K. Joshi, B. L. Webber, \& I. A. Sag (Eds.), Elements of discourse understanding (pp. 1063). Cambridge: Cambridge University Press. 
Clark, H. H., \& Schaefer, E. F. (1989). Contributing to discourse. Cognitive Science, 13, 259294. doi:10.1016/0364-0213(89)90008-6

Clark, H. H., \& Wilkes-Gibbs, D. (1986). Referring as a collaborative process. Cognition, 22, 1-39. doi:10.1016/0010-0277(86)90010-7

Gibbs, R. W. (1986). Comprehension and memory for nonliteral utterances: The problem of sarcastic indirect requests. Acta Psychologica, 62, 41-57.

Hirst, W., \& Echterhoff, G. (2012). Remembering in conversations: The social sharing and reshaping of memories. Annual Review of Psychology, 63, 55-79. doi:10.1146/annurev-psych-120710-100340

Hjelmquist, E. (1984). Memory for conversations. Discourse Processes, 7, 321-336. doi:10.1080/01638538409544595

Holtgraves, T., Srull, T. K., \& Socall, D. (1989). Conversation memory: The effects of speaker status on memory for the assertiveness of conversation remarks. Journal of Personality and Social Psychology, 56, 149-160.

Horton, W. S. (2008). A memory-based approach to common ground and audience design. In I. Kecskes (Ed.), Intention, common ground, and the egocentric speaker-hearer (pp. 189-222). Berlin/New York: Mouton de Gruyter.

Horton, W. S., \& Gerrig, R. J. (2005). Conversational common ground and memory processes in language production. Discourse Processes, 40, 1-35. doi: 10.1207/s15326950dp4001_1

Jaeger, T. F. (2008). Categorical data analysis: Away from ANOVAs (transformation or not) and towards logit mixed models. Journal of Memory and Language, 59, 434-446. doi:10.1016/j.jml.2007.11.007

Jarvella, R. J., \& Collas, J. G. (1974). Memory for the intentions of sentences. Memory and Cognition, 2, 185-188. doi:10.3758/BF03197513 
Keenan, J.M., MacWhinney, B., \& Mayhew, D. (1977). Pragmatics in memory: A study of natural conversation. Journal of Verbal Learning and Verbal Behavior, 16, 549-560.

Keselman, H. J., Algina, J., Kowalchuk, R. K., \& Wolfinger, R. D. (1999). The analysis of repeated measurements: A comparison of mixed-model satterthwaite $\mathrm{f}$ tests and a nonpooled adjusted degrees of freedom multivariate test. Communications in Statistics - Theory and Methods, 28, 2967-2999. doi: 10.1080/03610929908832460

* Kiernan, K., Tao, J., \& Gibbs, P. (2012). Tips and strategies for mixed modelling with SAS/STAT procedures. Paper presented at the 2012 SAS Global Forum, Orlando, FL.

Knutsen, D., \& Le Bigot, L. (2012). Managing dialogue: How information availability affects collaborative reference production. Journal of Memory and Language, 67, 326-341. doi:10.1016/j.jml.2012.06.001

MacLeod, C. M. (2011). I said, you said: The production effect gets personal. Psychonomic Bulletin and Review, 18, 1197-1202. doi:10.3758/s13423-011-0168-8

MacWhinney, B., Keenan, J. M., \& Reinke, P. (1982). The role of arousal in memory for conversation. Memory and Cognition, 10, 308-317.

Marsh, E. J. (2007). Retelling is not the same as recalling: Implications for memory. Current Directions in Psychological Science, 16, 16-20. doi: 10.1111/j.14678721.2007.00467.xPasupathi, M., \& Hoyt, T. (2010). Silence and the shaping of memory: How distracted listeners affect speakers' subsequent recall of a computer game experience. Memory, 18, 159-169. doi:10.1080/09658210902992917

Rajaram, S. (2011). Collaboration both hurts and helps memory: A cognitive perspective. Current Directions in Psychological Science, 20, 76-81. doi:10.1177/0963721411403251 
Samp, J. A., \& Humphreys, L. R. (2007). “I said what?” Partner familiarity, resistance, and the accuracy of conversational recall. Communication Monographs, 74, 561-581. doi:10.1080/03637750701716610

Stafford, L., Burggraf, C. S., \& Sharkey, W. E. (1987). Conversational memory: The effects of time, recall, mode, and memory expectancies on remembrances of natural conversations. Human Communication Research, 14, 203-229. doi:10.1111/j.14682958.1987.tb00127.x

Stafford, L., \& Daly, J. A. (1984). Conversational memory: The effects of recall mode and memory expectancies on remembrances of natural conversations. Human Communication Research, 10, 379-402.doi: 10.1111/j.1468-2958.1984.tb00024.x

Wilkes-Gibbs, D., \& Clark, H. H. (1992). Coordinating beliefs in conversation. Journal of Memory and Language, 31, 183-194. doi:10.1016/0749-596X(92)90010-U 


\section{Appendix A: Dialogue example and coding sample}

First, references were coded for presentation: a reference was coded as presented when it was produced at least once in the dyad (e.g., ST01 in Table A1). Because no hypothesis was formulated concerning presentation, no analysis was performed including this variable. Each presented reference was then coded as accepted. The evidence produced by the participant who did not present the reference (i.e., the addressee at the time of presentation) between the moment when a reference was presented and the moment when the initiator of the reference produced another reference was used to code for acceptance type. If the presented reference was repeated by the addressee, it was coded as accepted through verbatim repetition (e.g., ST03). If the landmark mentioned was re-referred to using a pronoun or a zero anaphor, the reference was coded as accepted anaphorically (e.g., ST07). If the landmark mentioned was not re-referred to, the reference was coded as accepted non-explicitly (e.g., the reference "Saint George Square" in the example). Finally, occurrences of reference production which could not be counted as presentations or acceptances were coded as reuses (e.g., ST08); the only criterion was that reuse had to occur in a speech turn preceded by a minimum of two speech turns during which the reference was not produced (see ST10). Each reused reference was coded for reuse by self and for reused by other from each participant's point of view (see Table A2). For instance, if a reference was reused by Partner A but not by Partner B, it was coded as reused by self and as non-reused by other from Partner A's point of view, and as non-reused by self and as reused by other from Partner B's point of view. An annotated dialogue extract can be found in Table A1; the purpose of Table A2 is to show how the different coding levels were represented in the datasheet used to perform the statistical analyses. 
Table A1

Annotated dialogue extract.

\begin{tabular}{|c|c|c|c|c|}
\hline $\begin{array}{l}\text { Speech } \\
\text { turn }\end{array}$ & Dyad & Speaker & $\begin{array}{l}\text { Utterance content (English } \\
\text { Translation) }\end{array}$ & Comments \\
\hline 01 & D01 & A & ok you start & \\
\hline 02 & D01 & B & $\begin{array}{l}\text { well what I suggest is that she goes } \\
\text { to the museum }\end{array}$ & $\begin{array}{l}\text { A reference to the museum is produced for the } \\
\text { first time. }\end{array}$ \\
\hline 03 & D01 & A & to the museum & $\begin{array}{l}\text { The participant who did not present the } \\
\text { reference "the museum" repeats this reference } \\
\text { before its initiator produces another reference. }\end{array}$ \\
\hline
\end{tabular}

References to Jean Jaurès Alley and Saint Antoine Street to go to the museum

Antoine Street are produced for the first time.

The reference to the museum is not coded as reused, as it was produced in the previous speech turn.

Level of coding

"the museum" $\rightarrow$ presented

"the museum" $\rightarrow$ accepted through verbatim repetition

"Jean Jaurès Alley" $\rightarrow$ presented

"Saint Antoine Street" $\rightarrow$ presented

The references to Jean Jaurès Alley and to Saint Antoine Street are not coded as reused, as they were mentioned in the second previous speech turn.

07 D01 A yes right I can see it

The participant who did not present the reference "Saint Antoine Street" uses a pronoun to re-refer to this landmark before its initiator produces another reference.
"Saint Antoine Street" $\rightarrow$ accepted anaphorically 
at the bottom of Saint Antoine Street you mean and there's a museum yeah

11 D01 A hm on my map I have Wilson's Square

12 D01 B hm alright
The reference to the museum is reused in a speech turn preceded by two speech turns in which it is not mentioned.

The reference to Saint Antoine Street is reused in a speech turn preceded by two speech turns in which it is not mentioned.

A reference to Saint George's Square is produced for the first time.

The reference to the museum is not coded as reused, as it was produced in the previous speech turn.

A reference to Wilson's square is produced for the first time.

A zero anaphor ("hm alright" in fact meaning "hm alright [you have Wilson's Square]) is used to re-refer to a reference before its initiator produces another reference.
"The museum" $\rightarrow$ reused

"Saint Antoine Street" $\rightarrow$ reused

"Saint George's Square" $\rightarrow$ presented

"Wilson's Square" $\rightarrow$ presented

"Wilson's Square" $\rightarrow$ accepted anaphorically

Table A2

Coding example.

\begin{tabular}{|c|c|c|c|c|c|c|}
\hline Dyad & Speaker & Reference & Acceptance type & Reuse in the dyad & Reuse by self & Reuse by other \\
\hline D01 & A & Museum & Verbatim repetition & Yes & No & Yes \\
\hline
\end{tabular}




\begin{tabular}{lllllll} 
D01 & B & Museum & Verbatim repetition & Yes & Yes & No \\
D01 & A & Jean Jaurès Alley & Non-explicit & No & No & No \\
D01 & B & Jean Jaurès Alley & Non-explicit & No & No & No \\
& & Saint Antoine Street & Anaphoric & Yes & Yes & No \\
D01 & A & Saint Antoine Street & Anaphoric & Yes & No & Yes \\
D01 & B & Saint George's Square & Non-explicit & No & No & No \\
D01 & A & Saint George's Square & Non-explicit & No & No & No \\
D01 & B & Anaphoric & No & No & No \\
D01 & A & Wilson's Square & Anaphoric & No & No & No \\
D01 & B & Wilson's Square & Anapo & & & \\
\hline
\end{tabular}




\section{Appendix B: Detail of the random effects structures used in the statistical analyses}

As specified in the main text, a logistic mixed model was used to analyze the data. One advantage of mixed models is that they allow considering both fixed effects (i.e., IVs) and random effects. There are two different kinds of random effects: random intercepts and random slopes. Random intercepts are used to account for potential variability across analysis units (e.g., participants, items). Random slopes are used to account for analysis units potentially differing in their sensitivity to the within-unit fixed effects.

Mixed models should include the maximal random effects structure justified by the experimental design (Barr, Levy, Scheepers, \& Tily, 2013). However, doing so might cause convergence problems; that is, the models might fail to find an appropriate solution within the specified number of iterations. Increasing the number of iterations does not always help solving such problems. Importantly, however, there are different kinds of convergence problems, one of which can be solved by adjusting the specified random effects structure. Specifically, this convergence problem concerns the G matrix and occurs when the variation associated with at least one of the random effects (intercept or slope) specified is either null or negative. For instance, if a researcher includes by-participant random intercepts in a mixed model but that the variation associated with participants is not statistically significant, doing so might cause the G matrix to fail to converge. In such cases, an appropriate solution consists in identifying the effect(s) causing the convergence problems and removing it or them from the model (Kiernan, Tao, \& Gibbs, 2012). Doing so does not affect the output of the analysis. In this study, the data were analyzed using the GLIMMIX procedure in SAS 9.4 using a variance components variance-covariance matrix. Because the data included a hierarchical structure (i.e., participants were nested in dyads), the initial model used included by-dyad, byparticipant and by-item (i.e., landmark) random intercepts. It also included by-participant random slopes corresponding to the IVs. However, this random effects structure caused G- 
matrix convergence problems, suggesting that at least one of the random effects did not significantly contribute to the model. We thus removed the random intercept and slopes causing the convergence failures; our main concern was to preserve the by-participant random intercepts, as the basic analysis unit was the participant, and the by-item random intercepts, as some landmarks might have been easier to remember than others (this also allowed controlling for potential primacy and recency effects). This led us to remove the by-dyad random intercepts, the by-participants random slopes corresponding to the Landmark Status and Reuse by Self IVs and the by-participant random slopes corresponding to the Reuse by Self * Reuse by Other interaction. Thus, the model reported in the main paper included byparticipant and by-item random intercepts and by-participant random slopes corresponding to Acceptance Type and Reuse by Other. Nonetheless, we hereafter report the covariance parameter estimates, the $F$ values and the model parameters from the full random effects structure model to ensure transparency. Note that the pattern of results reported in the paper and the pattern of results reported below are identical.

\section{Table B1}

Covariance parameter estimates, tests of fixed effects and model parameters using the full random effects structure model

\begin{tabular}{llll}
\hline $\begin{array}{l}\text { Covariance parameter estimates } \\
\text { Subject }\end{array}$ & Parameter & Estimate & Standard Error \\
\hline Dyad & Intercept & 0 & \\
Participant & Intercept & 0.34 & 0.12 \\
Participant & Landmark Status & 0 & \\
Participant & Acceptance Type & 0.02 & 0.07 \\
Participant & Reuse by Self & 0 & \\
Participant & Reuse by Other & 0.08 & 0.08 \\
Participant & Reuse by Self * Reuse by Other & 0 & \\
Item & Intercept & 0.51 & 0.13 \\
\hline
\end{tabular}




\begin{tabular}{lllll} 
Effect & Num DF & Den DF & $F$ value & $p$ value \\
\hline Landmark Status & 2 & 2076 & 25.96 & $<.001$ \\
Acceptance Type & 2 & 124 & 2.60 & .079 \\
Reuse by self & 1 & 2076 & 59.33 & $<.001$ \\
Reuse by other & 1 & 90.43 & 48.60 & $<.001$ \\
R. by self $*$ R. by other & 1 & 2076 & 12.62 & $<.001$ \\
\hline
\end{tabular}

\begin{tabular}{|c|c|c|c|c|c|}
\hline $\begin{array}{l}\text { Model parameters } \\
\text { Effect }\end{array}$ & Estimate & Standard Error & DF & $t$ value & $p$ value \\
\hline Intercept & -0.61 & 0.21 & 400 & -2.84 & .005 \\
\hline $\begin{array}{l}\text { Status: privileged-to- } \\
\text { other }\end{array}$ & -0.72 & 0.14 & 2076 & -5.26 & $<.001$ \\
\hline Status: privileged-to-self & 0.32 & 0.14 & 2076 & 2.31 & .021 \\
\hline Status: shared & 0 & & & & \\
\hline Acc. Type: Anaphoric & 0.24 & 0.16 & 137 & 1.48 & .142 \\
\hline Acc. Type: Verbatim & 0.43 & 0.19 & 223 & 2.28 & .024 \\
\hline Acc. Type: Non-explicit & 0 & & & & \\
\hline Reuse by self: yes & 1.37 & 0.15 & 2076 & 8.70 & $<.001$ \\
\hline Reuse by self: no & 0 & & & & \\
\hline Reuse by other: yes & 1.38 & 0.16 & 166 & 8.40 & $<.001$ \\
\hline Reuse by other: no & 0 & & & & \\
\hline R. self: yes, R. other: yes & -0.82 & 0.23 & 2076 & -3.55 & $<.001$ \\
\hline R. self: yes, R. other: no & 0 & & & & \\
\hline R. self: no, R. other: yes & 0 & & & & \\
\hline R. self: no, R. other: no & 0 & & & & \\
\hline
\end{tabular}

Note. Estimate values set to 0 correspond to the baselines used in the analysis. 